\title{
Impacto na saúde mental de crianças vítimas de abuso sexual
}

\author{
Impact on the mental health of children victims of sexual abuse
}

\section{Ana Paula Jesus da Silva', Aislan José de Oliveira ${ }^{2}$ e Fernanda Ellen dos Santos ${ }^{3}$}

Resumo: $O$ presente trabalho abrange a complexidade da conceituação do abuso sexual. A exploração do conteúdo apresentado possibilita o conhecimento sobre o desencadeamento da sexualização traumática das vítimas violentadas, além de sentimento de culpa e efeitos severos para lidar com relações. Com o intuito de compreender a influência relacionada a este trauma no desenvolvimento da criança e do adolescente, o objetivo da pesquisa foi evidenciar a gravidade desta agressão. Trata-se de uma pesquisa de abordagem qualitativa, descritiva e exploratória de revisão de literatura narrativa. Foram consultadas as bases de dados Scielo, Pepsic, Biblioteca Virtual em Saúde e Google Acadêmico, com as palavras-chaves: abuso sexual, infância, adolescência e saúde mental, considerando os artigos produzidos entre 2010 até 2019, além de livros, monografias e dissertações de mestrado. O estudo aponta as definições dos possíveis transtornos psicológicos que podem ser gerados a longo prazo, contribuindo para o conhecimento de forma ampla. Da mesma forma, discorre a respeito do efeito negativo na saúde mental das crianças e adolescentes vítimas deste abuso, referindo-se à qualidade de vida ao apontar o impacto emocional, psicológico, comportamental, social e cognitivo.

Palavras-chave: Crianças; Transtornos psicológicos; Abuso.

\begin{abstract}
The present study covers the complexity of conceptualizing sexual abuse. The exploration of the content presented allows the knowledge about the triggering of traumatic sexualization of raped victims, in addition to feelings of guilt and severe effects to deal with relationships. In order to understand the influence related to this trauma on the development of children and adolescents, the objective of the research is to highlight the seriousness of this aggression. This is a qualitative, descriptive and exploratory approach research of narrative literature review. Scielo, Pepsic, Virtual Health Library and Google Academic databases were consulted, with the keywords: sexual abuse, childhood, adolescence and mental health, considering the articles produced between 2010 and 2019, as well as books, monographs and master's dissertations. The study points out the definitions of possible psychological disorders that can be generated in the long term, contributing to the knowledge in a broad way. Likewise, it discusses the negative effect on mental health of children and adolescents victims of this abuse, referring to the quality of life by pointing out the emotional, psychological, behavioral, social and cognitive impact.
\end{abstract}

Keywords: Children; Psychological disorders; Abuse.

\footnotetext{
1 Psicóloga. Mestre. Docente no Centro Universitário Campos de Andrade - UNIANDRADE. E-mail anapaullajsilva@hotmail.com

2 Psicólogo. Mestre. Docente no Centro Universitário Campos de Andrade - UNIANDRADE. E-mail aislan_jo@hotmail.com

${ }^{3}$ Estudante do Curso de Psicologia do Centro Universitário Campos de Andrade - UNIANDRADE. E-mail fer.ellen@hotmail.com
} 


\section{Introdução}

Ao falar sobre abuso sexual há um senso comum enraizado com o foco apenas na violência com penetração envolvendo órgãos genitais, anulando os outros fatores que caracterizam tal fenômeno, o que ocasiona uma falta de compreensão real do assunto.

0 abuso sexual se trata de um fenômeno de grande ocorrência no escopo da violência contra crianças e adolescentes, uma vez que se configura por diversas práticas sexuais, tais como manipulação da genitália, pornografia, exibicionismo, assédio sexual, estupro, incesto e prostituição infantil (Serafim, Saffi, Rigonatti, Casoy \& Barros, 2009).

Infelizmente, a sociedade tende a minimizar os efeitos de abusos sexuais que não deixam marcas físicas, como se fossem menos importantes do que os que deixam hematomas. Assim, poucos estudos nacionais tratam da questão do abuso sexual com crianças e adolescentes fazendo uso de conceitos mais amplos, o que dificulta a compreensão do fenômeno com todas as suas peculiaridades, distorce estatísticas e limita políticas de intervenção adaptadas à realidade brasileira (Borges \& Delli, 2008).

Por ser uma experiência extremamente aversiva, 0 abuso sexual infantil e/ou na adolescência é considerado um importante fator de risco para vários problemas de saúde na infância e na vida adulta (Fergusson, Boden \& Horwood, 2008). Portanto, é de suma importância o trabalho multidisciplinar e intersetorial que envolva ações integradas de cuidado e atenção à saúde e proteção às crianças e adolescentes submetidas a situações de violência (Araújo, 2002).

Com o objetivo de apresentar conhecimento a respeito do impacto na saúde mental das crianças e adolescentes vítimas de abuso sexual, o presente estudo consiste em uma pesquisa de revisão de literatura narrativa, a fim de relatar consequências negativas na saúde emocional, psicológica, cognitiva e social das vítimas.

\section{Revisão de literatura}

A literatura define 0 abuso sexual como um fenômeno complexo que abrange vários recursos psicológicos e dimensões contextuais que contribuem para uma variedade de demonstrações comportamentais. Este tipo de abuso pode ser violento ou com menos violência, porém, íntimo, forçado ou codgido, que envolve penetração, atos não penetrativos e a assimetria de idade (Brackenridge, Bishopp, Moussali \& Taap, 2008).

Considerando que não há uma única descrição do que éo abuso sexual, a literatura aponta para algumas definições que apresentam aspectos em comum: 0 abuso sexual pode ser definido como um ato sexual sobre uma criança que não tem idade ou maturidade para o consentimento do mesmo, quando um indivíduo adulto ou um indivíduo menor de idade, porém fisicamente maior que a criança, exercendo uma posição de poder, envolve-se ou permite a aproximação dessa criança numa atividade de natureza sexual em que o objetivo é a gratificação sexual da pessoa adulta (Hartill, 2009).

№ Brasil, 83,2\% das vítimas de abuso sexual são do sexo feminino e 39,5\% ocorrem nas idades entre 10 e 14 anos. Em relação à etnia, os resultados do Comitê Nacional de Combateà Violência Sexual contra Crianças e Adolescentes também relatam maior incidência de abuso sexual nas crianças e adolescentes caucasianos (Adami, Chehab, Daboin, Figueiredo, Paiva \& Reato, 2017).
Aidade, o tipo de abuso (intrafamiliar ou extrafamiliar), o medo de consequências negativas e a responsabilidade frente ao abuso estão relacionados com o período que as vítimas levam para revelar a violência sexual (Goodman-Brown et. al., 2003). E, ao longo da situação, as vítimas estão sujeitas ao desenvolvimento de prejuízos em sua saúde mental, podendo diversificar devido às suas características pessoais e ao apoio social e/ou afetivo recebido. Os efeitos negativos podem variar entre os danos cognitivos, emocionais e comportamentais, refletindo em seu desempenho social, escolar e qualidade de vida (Cohen, Mannarino, \& Rogal, 2001; Jonzon \& Lindblad, 2004).

0 abuso ocorre quando um indivíduo reúne as condições de superioridade (idade, força, posição social ou econômica, inteligência e autoridade) e exerce 0 ato de causar dano físico, psicológico ou sexual, sem a vítima 0 consentir, contrariando a sua vontade, geralmente obtido por indução ou sedução enganosa (Brackenridge et al., 2008).

A violência sexual contra crianças e adolescentes, segundo Vaz (2001), ocorre muitas vezes no meio familiar ou em locais próximos, como vizinhança ou casa de parentes. A violência, na maior parte dos casos, não é denunciada e há a omissão de parentes ou conhecidos quanto ao crime cometido. Tal violência deixa "feridas afetivas"na criança que não são cicatrizadas, uma vez que 0 ato é praticado por al guém que a criança confia (Romaro \& Capitão, 2007).

Ainda que uma criança não apresente sintomas externos ou aparentemente sejam de pouca relevância, isto não quer dizer que ela não sofra ou não tenha prejuízos com os efeitos dessa experiência negativa. A vítima de abuso sexual pode apresentar um sofrimento emocional muito intenso. Além disso, suas consequências podem estar ainda latentes e talvez se manifestem posteriormente, frente à resolução de uma crise evolutiva ou situacional e frente ao estresse. Dessa forma, uma criança abusada deve ser considerada uma criança em situação de risco (Amazarray \& Koller, 1998).

0 envolvimento de crianças e adolescentes em interações sexuais inadequadas à sua faixa etária poderá desencadear em uma sexualização traumática (Finkelhor \& Browne, 1985). Contudo, entende-se que o impacto psicológico sofrido pelas vítimas pode ser de diversas naturezas e as alterações podem ser apresentadas em curto e/ou longo prazo a partir dos episódios abusivos (Silva, Gava, \& Dell'Aglio, 2013).

É possível observar na literatura a existente concordância entre os especialistas em reconhecer que a criança vítima de abuso e de violência sexual corre o risco de uma psicopatologia grave, que perturba sua evolução psicológica, afetiva e sexual (Romaro \& Capitão, 2007). Além disso, sentimento de culpa é corriqueiro entre crianças e adolescentes sexualmente abusados, sendo um dos efeitos emocionais mais severos que resultam da interação abusiva, especialmente se esta foi incestuosa e durou por muito tempo. Soma-se ao sentimento de culpa, o dano secundário da estigmatização, devido à acusação por parte dos pais e da família (Furniss, 1993).

As consequências decorrentes do abuso sexual variam desde efeitos mínimos até problemas mais graves, com repercussões sociais, emocionais e/ou psiquiátricas - como depressão, transtornos de ansiedade (entre os quais, o Transtorno de Estresse Pós-Traumático), transtornos alimentares, transtornos dissociativos, Transtorno de Déficit de Atenção/Hiperatividade e, até mesmo, Transtorno da Personalidade Borderline (Saywitz, Mannarino, Berliner \& Cohen, 2000; Collin-Vézina \& Hébert, 2005). Além disso, acrescenta-se também a manifestação de sintomas como: tristeza, ideação suicida, medo exagerado de adultos, comportamento sexual avançado para a idade, 
masturbação frequente e/ou pública, baixa autoestima, abuso de substâncias químicas, sonolência, enurese, encoprese, tiques e manias, isolamento social, dificuldades de aprendizagem, irritabilidade, entre outros (Polanczyck et al., 2003; Pfeiffer \& Salvagni, 2005).

Pesquisadores concordam que o abuso sexual infantil é facilitador para 0 aparecimento de psicopatologias graves, prejudicando a evolução psicológica, afetiva e social da vítima. Os efeitos do abuso na infância podem se manifestar de várias maneiras, em qualquer idade da vida (Romaro \& (apitão, 2007).

Quanto ao impacto na vivência das crianças e adolescentes, a literatura ressalta que as possíveis alterações na saúde mental e a futura adaptação social das vítimas estão diretamente relacionadas às características de personalidade de cada vítima, bem como ao tipo de violência sofrida e à capacidade de reação diante de fatos geradores de estresse (Zavaschi, Satler, Poester, Vargas, Piazenski \& Rohde, 2002). Além disso, a relação com a ocorrência de delitos na esfera sexual também é uma consequência desta experiência negativa. Há uma frequência notável de jovens delinquentes mantidos em instituições penais com histórico de abuso, negligência e experiências traumatizantes (Gover \& Mackenzie, 2003).

0 processo de revelação do abuso sexual constitui-se como um elemento chave para a compreensão dos desdobramentos que esta violência pode gerar na vida das vítimas e suas famílias. A credibilidade que uma vítima recebe quanto ao seu relato, bem como a existência de uma rede de apoio social e afetiva - interna ou externa à família - é central para um prognóstico mais positivo e rotativo para a vítima de violência sexual, uma vez que funciona como um importante mediador do impacto da violência sexual sobre a vítima (Habigzang et al., 2006; Santos \& Dell'Aglio, 2013).

0 processo de revelação ainda é dificultado pelo pacto de silêncio (Furniss, 1993), que se estabelece nos casos de abuso sexual contra crianças, um entrave para que este seja impedido e os agressores punidos. A falta de punição e a recorrência do ato sexual violento podem, muitas vezes, levar a criança à morte ou deixar graves sequelas físicas e psíquicas (Romaro \& (apitão, 2007).

A efetividade dos tratamentos psicoterápicos para crianças e adolescentes vítimas de abuso sexual tem sido investigada, a fim de disponibilizar as melhores estratégias de tratamento (Cohen, Mannarino, \& Knudsen, 2005; Habigzang et. al., 2009; Habigzang, Damásio, \& Koller, 2013). Os profissionais que atuam junto a crianças e adolescentes necessitam conhecer as características e os fatores de risco associados às situações abusivas, ressaltando-se a importância da compreensão da própria definição de abuso e maus-tratos e das consequências desses episódios no desenvolvimento infanto-juvenil e psicopatológico (Polanczyck et. al., 2003).

\section{Método}

Trata-se de uma pesquisa de abordagem qualitativa, descritiva e exploratória de revisão de literatura narrativa, foram consultadas as bases de dados Scielo, Pepsic, BVS e Google Acadêmico, com as palavras-chaves: abuso sexual, infância, adolescência e saúde mental. A fim de aprofundar o conhecimento sobre o tema e torna-lo explícito, realizou-se uma pesquisa bibliográfica com base de publicações em artigos e redes eletrônicas, considerando os artigos produzidos entre 0 ano de 1985 até 2019, da língua portuguesa e inglesa, além de livros, teses e dissertações de mestrado de encontro ao tema pesquisado.

\section{Resultados e discussão}

Os resultados apontam para o desenvolvimento de transtornos psicológicos concebidos a longo prazo em crianças e adolescentes vítimas de abuso sexual, impactando negativamente em sua saúde mental e qualidade de vida. Um indivíduo normalmente não sai ileso de uma experiência traumática como 0 abuso sexual infantil. Normalmente al guns transtornos estão mais associados à experiência, os quais estão descritos brevemente a seguir.

\section{Transtorno Depressivo}

Sendo a alteração mais comum, o Transtorno Depressivo é um dos principais diagnósticos encontrados em vítimas de abuso sexual. O prejuízo no desenvolvimento acarretado pela depressão infantil pode ser em nível físico, cognitivo, psicomotor e psicossocial, afetando principalmente as habilidades necessárias para a aprendizagem (Fichtner, 1997). Portanto, as crianças depressivas têm dificuldades para entender explicações e concentrar-se, uma vez que quando o emocional está comprometido há interferências nas questões cognitivas (Barbosa \& Lucena, 1995). Isso explica a queda abrupta do rendimento escolar em crianças e adolescente vítimas de abuso sexual.

Os principais comportamentos que caracterizam a depressão infantil são: sintomas físicos (dores de cabeça e abdominais, fadiga e tontura), seguidos por ansiedade, fobias, agitação psicomotora ou hiperatividade, irritabilidade, diminuição do apetite, alteração do peso e com menor frequência a ocorrência de enurese e encoprese. Também é possível observar a fisionomia triste, comunicação deficiente, choro frequente, movimentos repetitivos e heteroagressividade na forma de comportamento agressivo e destrutivo, auto depreciação, distúrbios do sono, diminuição da socialização, modificação de atitudes em relação à escola, perda de energia habitual, tristeza, humor disfórico, retardo psicomotor, pesadelos, terror noturno, ansiedade de separação, diminuição da capacidade cognitiva e perda de interesse pelas atividades prazerosas dessa etapa da vida. As crianças deprimidas não conseguem rir, brincar ou brigar, por isso são consideradas enfermas, além disso, são tímidas e evitam estar com outras pessoas (Barbosa \& Lucena, 1995).

\section{Transtornos de Ansiedade}

Os Transtornos de Ansiedade também são comumente observados em vítimas de abuso sexual, muitas vezes associados à depressão. Crianças e adolescentes com Transtornos de Ansiedade tendem a exibir preocupações demasiadas com seu desempenho, perfeccionismo e insegurança (Costa, 2010). A preocupação excessiva é acompanhada por uma variedade de sintomas somáticos, o que pode sinalizar Transtorno de Ansiedade Generalizada (TAG) e causar comprometimento significativo no funcionamento social e ocupacional do indivíduo (Oliveira \& Souza, 2010).

De acordo com o DSM-V (Manual de Diagnóstico e Estatístico de Transtornos Mentais), o Transtorno de Estresse Pós-Traumático (TEPT) é considerado um distúrbio de ansiedade precipitado por um trauma, podendo ser diagnosticado conforme o período de duração, com a possibilidade de ser agudo, crônico ou de início tardio. Os sintomas apresentados de forma aguda têm a duração superior a um mês, mas inferior a três meses e, como crônico, se a duração é superior a esse período. Se o início dos sintomas ocorre em um período superior a seis meses após o evento estressor, especifica-se TEPT com início tardio (APA, 2014). 
As alterações estruturais e funcionais relacionadas ao TEPT são bastante complexas e precisam ser examinadas com cautela. 0 desenvolvimento e a gravidade de transtornos relacionados ao estresse podem estar condicionados à vulnerabilidade individual, a fatores ambientais e à idade em que ocorreu a situação estressante (Caminha, 2005). Pode-se afirmar que os tipos de manifestações apontados pelo transtorno de estresse pós-traumático, sejam eles de ordem psicológica, física, psicológica, com componente depressivo ou psicofisiológico, geram na criança dificuldades emocionais, cognitivas e comportamentais (Oliveira \& Santos, 2006).

\section{Transtornos Alimentares}

Há fatores de diferentes naturezas que contribuem para o aparecimento deste transtorno, como fatores biológicos, psicológicos, socioculturais, genéticos e familiares. As razões que ocasionam a sua manifestação são aquelas que aumentam as chances do seu aparecimento. Questões socioculturais junto a fatores genéticos podem tornar um indivíduo mais predisposto a desenvolver um transtorno alimentar. No entanto, experiências sexuais abusivas em idade precoce aumentam a vulnerabilidade a transtornos psiquiátricos em geral, 0 que seria um fator de risco para a anorexia e a bulimia (Morgan et. al., 2002).

A criança vítima de abuso pode passar a depreciar seu corpo, sentir repulsa pelo mesmo e ter comportamentos autodestrutivos. Vale ressaltar que a autoestima baixa, associada também à depressão e à ansiedade podem culminar em estratégias negativas para manejo dos sentimentos que vêm à tona. Deixar de alimentar-se ou ter comportamentos purgativos para manejo da compulsão direcionada à comida podem ser formas de sinalizar a insatisfação com o que o corpo representa.

\section{Transtorno Dissociativo de Identidade (TDI)}

0 Transtorno Dissociativo de Identidade (TDI) é um tipo de Transtorno Dissociativo caracterizado por dois ou mais estados de personalidade que se apresentam de modo alternado, sendo eles os chamados alter, autoestados ou identidades (Spiegel, 2015). No que diz respeito ao distúrbio propriamente dito, o TDI ou personalidade múltipla é de origem psicorreativa, geralmente súbito e transitório, significando um quadro que surge como reação a alguma vivência traumática. Este transtorno é caracterizado por perturbações das funções integrativas da identidade, memória e consciência (Putnam, 1989). Destaca-se que o TDI engloba a incapacidade de lembrar informações pessoais importantes, eventos diários e eventos traumáticos ou estressantes, os quais não seriam normalmente esquecidos (Schreiber, 1973; Spira \& Yalom, 1996; Dell \& O'neil, 2009; Faria, 2013; Spiegel, 2015;).

OTDI pode ser resultado de algum desequilíbrio que afeta a personalidade, como os traumas. Além disso, indivíduos com TDI relatam frequentemente experiências de severo abuso físico e sexual, especialmente na infância (Putnam, 1989).

\section{Transtorno da Personalidade Borderline (TPB)}

De acordo com o DSM-V, para o diagnóstico do TPB, o indivíduo deve apresentar cinco ou mais das seguintes características: padrão de instabilidade de relacionamento afetivo, interpessoal e autoimagem, além de grande impulsividade que começa no início da idade adulta e se apresenta em variados contextos; os indivíduos se esforçam de modo exagerado para não serem abandonados real ou imaginariamente, são sensiveis às circunstâncias ambientais, temendo abandono e apresentando raiva intensa ou dificuldade em controlá-la, mesmo em separações reais por tempos limitados; padrão de relacionamento interpessoal instável e intenso variando da idealização à desvalorização (APA, 2014).

No Transtorno de Personalidade Borderline existe um distúrbio de identidade caracterizado por autoimagem ou sentimento de self acentuado e persistentemente instável; impulsividade com relação a gastos financeiros, abuso de substâncias, sexo inseguro, direção imprudente e comer compulsivamente; comportamentos suicidas ou automutilação; instabilidade afetiva causada pela reatividade do humor; sentimento crônico de vazio e ideação paranoide transitória relacionada ao estresse ou a sintomas graves de dissociação (APA, 2014).

\section{Conclusão}

A discussão sobre o conceito de abuso sexual infantil trouxe à tona uma reflexão importante sobre o que pode ser considerado abuso e qual o impacto do mesmo na saúde mental das vítimas. A pesquisa realizada apontou os principais transtornos psicológicos desenvolvidos a longo prazo e relacionados a experiência traumática em tela.

Sabendo do prognóstico ruim de uma experiência como o abuso sexual infantil, é fundamental discorrer a respeito do acompanhamento e tratamento das crianças e adolescentes vítimas deste tipo de violência. É importante ressaltar que o profissional precisa estar capacitado para lidar com tal problemática a fim de desenvolver com a vítima uma relação de confiança, de modo que a mesma se sinta segura e confortável no momento da escuta. 0 impacto de uma experiência traumática depende da interpretação feita pela vítima posteriormente aos acontecimentos e essa interpretação depende da qualidade do apoio que a vítima recebe. É importante saber como uma pessoa processa a experiência que teve, suas atribuições de responsabilidade, percepção de controle e autoestima.

De acordo com os apontamentos levantados a respeito do processo de revelação do abuso sexual, constatou-se ser importante a habilidade do profissional ouvinte ter empatia e permitir as expressões espontâneas da vítima durante a escuta, evitando métodos interrogatórios, julgamentos e atribuições de culpa.

Vale ressaltar que vítimas de maus-tratos na infância e/ou adolescência podem tornar-se multiplicadores de maus-tratos na vida adulta. A compreensão do papel de vítima permite o desenvolvimento de habilidades de autoproteção para a prevenção da revitimização mas, para isso, sinaliza-se a necessidade de um profissional psicólogo inserido nas comunidades (grupos de apoio à vítimas de abuso sexual, inserção em escolas com treinamento de professores, participação em grupos comunitários, centros de assistência social, grupos de orientação para pais), a fim de fornecer o apoio necessário para as vítimas em diversos contextos.

Para finalizar é importante ressaltar que somente a educação é capaz de levar conhecimento e principalmente proporcionar o entendimento de um fenômeno. Esse estudo traz como propósito a reflexão sobre as consequências do abuso sexual infantil a longo prazo e a importância de uma intervenção adequada. Mas, para isso, faz-se necessária a criação de políticas públicas que garantam serviços de qualidade e isso só será possível por meio de novas pesquisas neste campo de estudo. 
Ainda que o tema seja bastante discutido e as estatísticas sinalizem 0 crescimento de notificações de casos de crianças vítimas de violência, ainda são insipientes propostas de intervenção mais eficazes e políticas públicas que contemplem a dinâmica do fenômeno de uma forma mais ampla. Assim, são necessários mais estudos na área e pesquisas que fomentem a necessidade de programas de prevenção e intervenção que sejam capazes de amenizar o impacto do abuso sexual na saúde mental de crianças vítimas de abuso sexual.

\section{Referências}

Adami, F., Chehab, D., Daboin, G., E., B., Figueiredo, W., S., F., Paiva, S., L., A., M., \& Reato, N., F., L. (2017). Sexual abuse characteristics in Santo André, São Paulo, Brazil: from victims to aggressors, from diagnosis to treatment. JHum Growth Dev. 27(2). 228-234. https://repositorio.iscteiul.pt/bitstream/10071/20049/1/Master_Maria_Marques_Gama.pdf

Amazarray, M. R. \& Koller, S. H. (1998). Alguns aspectos observados no desenvolvimento de crianças vítimas de abuso sexual. Psicologia: Reflexão e Crítica. Porto Alegre, v. 11, n. 3. http://www.scielo.br/scielo.php?scrip$\mathrm{t}=$ sci_arttext\&pid=S010279721998000300014\&lng=en\&nrm=iso.

American Psychiatric Association. (2014). DSM-V - Manual Diagnóstico e Estatístico de Transtornos Mentais. Porto Alegre: Artmed.

Araújo M. F. (2002). Violência e abuso sexual na família. Psicologia em Estudo; 7(2):3-11. https://www.scielo.br/pdf/csc/v23n4/1413-8123csc-23-04-1019.pdf

Barbosa, G. A. \& Lucena, A. (1995). Depressão infantil. RNIA, 3(2), 23-30. http://www.psiquiatriainfantil.com.br/revista/edicoes/Ed_03_2/ in_07_07.pdf.

Borges, J. L. \& Delli Aglio, D. D. (2008). Abuso sexual infantil: indicadores de risco e consequências no desenvolvimento de crianças. Interam J Psicol, 42(3):528-36. https://www.scielo.br/pdf/rpc/v38n4/a06v38n4.pdf.

Brackenridge, C. H., Bishopp, D., Moussalli, S. \& Tapp, J. (2008). The characteristics of sexual abuse in sport: A multidimensional scaling analysis of events described in media reports. International Journal of Sport and Exercise Psychology. 6: 365-406. https://repositorio.iscteiul.pt/bitstream/10071/20049/1/Master_Maria_Marques_Gama.pdf

Caminha, R. M. (2005). Transtorno do Estresse Pós-Traumático (TEPT): da neurobiologia à terapia cognitiva. São Paulo: Casa do Psicólogo.

Castillo, A. R. G. \& et. al. (2000). Transtornos de Ansiedade. Rev. Bras. Psiquiatria. Retirado de https://www.scielo.br/pdf/rbp/v22s2/3791.pdf.

Cohen, J. A., Mannarino, A. P.\& Knudsen, K. (2005). Treating sexually abused children: One-year follow-up of a randomized controlled trial. Child Abuse \& Neglect, 29, 135-145. Retirado de http://dx.doi.org/10.1016/j. chiabu.2004.12.00.

Cohen, J. A., Mannarino, A. P. \& Rogal, S. (2001). Treatment practices for childhood posttraumatic stress disorder. Child Abuse \& Neglect, 25, 123-135. https://www.scielo.br/pdf/epsic/v13n3/a11v13n3.pdf.

Collin-Vézina, D. \& Hébert, M. (2005). Comparing dissociation and PTSD in sexually abused school-aged girls. Journal of Nervous and Mental Disease, 193(1), 47-52. https://www.scielo.br/pdf/ptp/v28n2/11.pdf.

Dell, P.F., \& O'neil, A. (2009). Dissociation and the Dissociative Disorders: DSM-V and Beyond. New York: Routledge.

Faria, M. A. (2013). Impacto do trauma e dissociação da consciência na personalidade múltipla. Brasília: Kiron.

Fergusson, D. M., Boden, J. M. \& Horwood, L. J. (2008). Exposure to childhood sexual and physical abuse and adjustment in early adulthood. Child Abuse Negl, 32(6):607-619. https://www.researchgate.net/publication/5290198_Exposure_to_childhood_sexual_and_physical_abuse_and_adjustment_in_early_adulthood.
Fichtner, N. (1997). Transtornos mentais da infância e da adolescência: Um enfoque desenvolvimental. Porto Alegre: Artmed.

Finkelhor, D. \& Browne, A. (1985). The traumatic impact of child sexual abuse: A conceptualization. American Journal of Orthopsychiatry, v. 55, n. 4, pp. 530-541. https://doi.apa.org/doiLanding?doi=10.1111\%2Fj.1939-0025.1985.tb02703.x.

Fowler, D. N. \& Chanmugam, A. (2007). A Critical Review of Quantitative Analyses of Children Exposed to Domestic Violence: Lessons for Practice and Research. Brief Treatment and (Crisis Intervention, 7(4), 322-344. http:// triggered.edina.clockss.org/ServeContent?url=http\%3A\%2F\%2Fbtci. stanford.clockss.org\%2Fcgi\%2Freprint\%2F7\%2F4\%2F322

Furniss, T. (1993). Abuso Sexual da Criança: Uma Abordagem Multidisciplinar. Porto Alegre: Artes Médicas.

Goodman-Brown, T, B., Edelstein, R. S., Goodman, G. S., Jones, D. P. H. \& Gordon, D. S. (2003). Why children tell: a model of children's disclosure of sexual abuse. Child Abuse \& Neglect, 27, 525-540. https://www. researchgate.net/publication/10783965_Why_children_tell_A_model_of_children's_disclosure_of_sexual_abuse.

Gover, R. \& Mackenzie, D. L. (2003). Child maltreatment and adjustment to juvenile correctional institutions. Criminal, Justice and Behavior; 30(3):37496. https://www.scielo.br/pdf/rpc/v38n4/a06v38n4.pdf.

Habigzang, L. F., Azevedo, G. A., Koller, S. H. \& Machado, P. X. (2006). Fatores de Risco e de Proteção na rede de atendimento a Crianças e Adolescentes Vitimas de Violência Sexual. https://www.scielo.br/pdf/prc/v19n3/a06v19n3.pdf.

Habigzang, L. F., Damásio, B. F. \& Koller, S. H. (2013). Impact evaluation of a cognitive behavioral group therapy model in brazilian sexually abused girls. Journal of Child Sexual Abuse, 22(2), 173-90. https://doi.org/10.1 080/10538712.2013.737445

Habigzang, L. F., Stroeher, F., Hatzenberguer, R., Cunha, R. C., Ramos, M. \& Koller, S. H. (2009). Grupo terapia cognitivo-comportamental para crianças e adolescentes vítimas de abuso sexual. Revista de Saúde Pública, 43, 70-78. https://www.scielo.br/pdf/rsp/v43s1/759.pdf.

Hartill, M. (2009). The Sexual Abuse of Boys in Organized Male Sports. Men and Masculinities. Vol. 12 (2). 225-249. https://repositorio.iscte-iul.pt/ bitstream/10071/20049/1/Master_Maria_Marques_Gama.pdf.

Jonzon, E. \& Lindblad, F. (2004). Disclosure, reactions and social support: findings from a sample of adult victims of child sexual abuse. Child Maltreatment, 9(2), 190-200. https://www.scielo.br/pdf/epsic/v13n3/ a11v13n3.pdf.

Morgan, C. M., Vecchiatti, I. R. \& Negrao, A. B. (2002). Etiologia dos transtornos alimentares: aspectos biológicos, psicológicos e socioculturais. http:// dx.doi.org/10.1590/S1516-44462002000700005.

Oliveira, L. H. \& Santos, C. S. S. (2006). As diferentes manifestações do transtorno de estresse pós-traumático (TEPT) em crianças vítimas de abuso sexual. Rev. SBPH, Rio de Janeiro, v. 9, n. 1, jun. http://pepsic.bvsalud. org/scielo.php?script=sci_arttext\&pid=\$151608582006000100004\&l$\mathrm{ng}=\mathrm{pt \& nrm}=\mathrm{is}$.

Oliveira, M. A. M. \& Sousa, W. P. S. (2010). 0 uso da Terapia Cognitiva no tratamento do Transtorno de Ansiedade Generalizada. Anais da XVIII Semana de Humanidades. Universidade Federal do Rio Grande do Norte. Natal.

Pfeiffer, L. \& Salvagni, E. P. (2005). Visão atual do abuso sexual na infância e adolescência.Jornal de Pediatria, 81(5), 197-204. https://www.scielo.br/ pdf/jped/v81n5s0/v81n5Sa10.pdf.

Polanczyck, G., Zavaschi, M. L., Benetti, S. P. C., Zenker, R. \& Gammerman, P. (2003). Violência sexual e sua prevalência em adolescentes de Porto Alegre, Brasil. Revista de Saúde Pública, 37(1), 8-14. https://www.scielo. $\mathrm{br} / \mathrm{pdf} / \mathrm{rsp} / \mathrm{v37n1/13539.pdf.}$

Putnam, F.W. (1989). Diagnosis and treatment of multiple personality disorder. New York: Guilford.

Romaro, R. A. \& Capitão, C. G. (2007). As faces da violência: aproximações, pesquisas, reflexões. São Paulo: Vetor. 
Santos, S. S., \& Dell'Aglio, D. D. (2013). 0 processo de revelação do abuso sexual na percepção de mães. Psicologia: Teoria e Prática, 15(1), 50-64. http://pepsic.bvsalud.org/pdf/ptp/v15n1/04.pdf.

Saywitz, K. J., Mannarino, A. P., Berliner, L. \& Cohen, J. A. (2000). Treatment for sexually abused children and adolescents. American Psychologist, 55(9), 1040-1049. https://revistaseletronicas.pucrs.br/ojs/index.php/ revistapsico/article/view/16718/12945.

Serafim A. P., Saffi F., Rigonatti S. P., Casoy I. \& Barros D.M. (2009). Perfil psicológico e comportamental de agressores sexuais de crianças. Rev Psiq Clinn., 36(3):101-11. https://www.scielo.br/pdf/rpc/v36n3/v36n3a04.pdf.

Silva, D. G., Gava, L. L. \& Dell'Aglio, D. D. (2013). Sintomas e quadros psicopatológicos em supostas vitimas de abuso sexual: uma visão a partir da psicologia positiva. Aletheia, 44(2), 235-244. http://pepsic.bvsalud.org/ pdf/aletheia/n40/n40a06.pdf.

Spiegel, D. (2015). Dissociative identity disorder. Merck Manuals. Merck \& Co., Inc.,Kenilworth, NJ, USA.

Spira, J. L., \& Yalom, I. D. (1996). Treating Dissociative Identity Disorder. San Francisco:Jossey-Bass Publishers.

Zavaschi M. L. S., Satler, F., Poester, D., Vargas, C. F., Piazensk,i R. \& Rohde, L. A. P. (2002). Association between childhood loss trauma and depression in adulthood. Rev Bras Psiquiatr., 24(4):189-95. https://www.scielo.br/ pdf/rbp/v24n4/12728.pdf. 